OPEN ACCESS

Edited by: George Broufas, Democritus University of Thrace,

Greece

Reviewed by: Javier Plasencia,

National Autonomous University of Mexico, Mexico Massimo Reverberi,

Sapienza University of Rome, Italy

*Correspondence:

Subbaiah Chalivendra schalivendra@agcenter.Isu.edu; subbaiahchalivendra@gmail.com

Specialty section:

This article was submitted to Plant Pathogen Interactions,

a section of the journal

Frontiers in Plant Science

Received: 24 May 2020

Accepted: 19 August 2020

Published: 23 September 2020

Citation:

Chalivendra S, Huang F, Busman M, Williams WP and Ham JH (2020) Low Aflatoxin Levels in Aspergillus flavus-

Resistant Maize Are Correlated With Increased Corn Earworm Damage and Enhanced Seed Fumonisin.

Front. Plant Sci. 11:565323. doi: 10.3389/fp/s.2020.565323

\section{Low Aflatoxin Levels in Aspergillus flavus-Resistant Maize Are Correlated With Increased Corn Earworm Damage and Enhanced Seed Fumonisin}

\author{
Subbaiah Chalivendra ${ }^{1 *}$, Fangneng Huang ${ }^{2}$, Mark Busman ${ }^{3}$, W. Paul Williams ${ }^{4}$ \\ and Jong Hyun Ham ${ }^{1}$
}

${ }^{1}$ Department of Plant Pathology and Crop Physiology, Louisiana State University AgCenter, Baton Rouge, LA, United States, 2 Department of Entomology, Louisiana State University AgCenter, Baton Rouge, LA, United States, ${ }^{3}$ Bacterial Foodborne Pathogens and Mycology Research Unit, USDA-ARS-NCAUR, Peoria, IL, United States, ${ }^{4}$ Corn Host Plant Resistance Research Unit, USDA-ARS, Mississippi State, MS, United States

Preharvest mycotoxin contamination of field-grown crops is influenced not only by the host genotype, but also by inoculum load, insect pressure and their confounding interactions with seasonal weather. In two different field trials, we observed a preference in the natural infestation of corn earworm (CEW; Helicoverpa zea Boddie) to specific maize (Zea mays L.) genotypes and investigated this observation. The field trials involved four maize lines with contrasting levels of resistance to Aspergillus flavus. The resistant lines had 7 to 14 -fold greater infested ears than the susceptible lines. Seed aflatoxin $B_{1}(\mathrm{AF})$ levels, in mock- and $A$. flavus-inoculated ears were consistent with genotype resistance to $A$. flavus, in that the resistant lines showed low levels of $A F(<30$ $\mathrm{ppb}$ ), whereas the susceptible lines had up to $500 \mathrm{ppb}$. On the other hand, CEW infestation showed a positive correlation with seed fumonisins (FUM) contamination by native Fusarium verticillioides strains. We inferred that the inverse trend in the correlation of AF and FUM with $H$. zea infestation may be due to a differential sensitivity of CEW to the two mycotoxins. This hypothesis was tested by toxin-feeding studies. $H$. zea larvae showed decreasing mass with increasing AF in the diet and incurred $>30 \%$ lethality at 250 ppb. In contrast, CEW was tolerant to fumonisin with no significant loss in larval mass even at $100 \mathrm{ppm}$, implicating the low seed aflatoxin content as a predominant factor for the prevalence of CEW infestation and the associated fumonisin contamination in A. flavus resistant maize lines. Further, delayed flowering of the two resistant maize lines might have contributed to the pervasive $H$. zea damage of these lines by providing young silk for egglaying. These results highlight the need for integrated strategies targeting mycotoxigenic fungi as well as their insect vectors for enhanced food safety.

Keywords: Maize (Zea mays), Aspergillus flavus, Fusarium verticillioides, ear rot, resistant and susceptible lines, corn earworm (Helicoverpa zea Boddie), aflatoxin toxicity, fumonisin tolerance 


\section{INTRODUCTION}

Besides causing crop damage and economic loss to the grower, mycotoxigenic fungi pose a serious risk to human and livestock health due to the contamination of commodities with carcinogenic and neurotoxic secondary metabolites known as mycotoxins. Aflatoxin $\mathrm{B}_{1}(\mathrm{AF})$ is the most dangerous among mycotoxins due to its very potent carcinogenicity. Aspergillus flavus, an opportunistic pathogen, is the predominant species that contaminates cereal and oil seed crops with AF. Although not as genotoxic as AF, fumonisins (FUM) are associated with esophageal cancer, particularly due to cytotoxicity of fumonisin $\mathrm{B}_{1}\left(\mathrm{FB}_{1}\right)$. They are also among the most common food- and feed-contaminating mycotoxins in many countries (Biomin, 2015; Munkvold et al., 2019). FUM are produced by Fusarium species, F. verticillioides (formerly known as $F$. moniliforme) being the predominant contaminant of commodities (Munkvold, 2003). A. flavus and F. verticillioides cause ear rots in maize (Zea mays L.), a globally important food, feed and fuel crop of high productivity. AF and FUM can be co-contaminants of commodities (Guo et al., 2017), particularly in high cancer-risk areas (Sun et al., 2011), and act synergistically on carcinogenesis (Lopez-garcia, 1998).

Aspergillus and Fusarium ear rots are more frequent in warmer and drier cropping seasons or a warmer and wetter weather combination at the time of harvest, and are often exacerbated by insect damage. Insect-vectored inoculum can breach the natural plant defense. The invasive methods of inoculation by chewing and piercing insects would bypass resistance mechanisms, such as remote defense signals triggered in the husk, silk or seed surface in response to natural infection via silk. Consequently, ear rot diseases are more common in the southern United States (US) and lowland tropics (Miller, 1994; reviewed in Cotty and Jaime-Garcia, 2007; Santiago et al., 2015). Among insect pests infesting maize, European Corn Borer (ECB) causes the most serious damage (Boyd and Bailey, 2001; Hutchison et al., 2010). It not only injures plants, exposing them to infection, but also vectors ear rot and stalk rot fungi, particularly $F$. verticillioides and $F$. graminearum (Widstrom, 1992). Extensive use of Bt (Bacillus thurigiensis crystal proteins-expressing) maize, with its high efficacy against ECB, has reduced overall ECB populations in the US (Hutchison et al., 2010). Maize pests previously considered as secondary to ECB are now taking its position (Bowers et al., 2014). Corn earworm [CEW; Helicoverpa zea (Boddie); formerly in the genus Heliothis] has become the most economically important pest in the southern US where nonfreezing winters are conducive for it to multiply by $4-7$ generations in a year. Resistance of this pest to a wide range of insecticides and to Bt maize has also been documented (Capinera, 2004; Dively et al., 2016; Kaur et al., 2019). Although CEW has multiple crop and weed hosts, maize is its preferred host (Johnson et al., 1975). Annual yield loss due to CEW ranges from $2-17 \%$ for field corn and up to $50 \%$ in sweetcorn in the southern US. A. flavus and F. verticillioides invade the seed through silk and are also vectored by CEW and other ear-infesting insects (Munkvold and White, 2016). F. verticillioides can grow also as an endophyte through root or stem infection, and is vectored by insects such as ECB that feed on vegetative tissues (Blacutt et al., 2018). In addition to facilitating fungal colonization, insect infestation can also enhance mycotoxin production in host tissues (Döll et al., 2013; Drakulic et al., 2015; Drakulic et al., 2016). Unlike a strong association observed in the case of FUM contamination (e.g., Smeltzer, 1959; Dowd, 2000; Mesterházy et al., 2012), seed AF levels were reported to be poorly correlated with CEW damage caused by either natural invasion (Ni et al., 2011; Cao et al., 2014) or manual infestation (Lillehoj et al., 1984). A metaanalysis of published work showed a 59\% reduction in the mean $\mathrm{FB}_{1}$ concentration in Bt maize compared to the non- $\mathrm{Bt}$ control (Cappelle, 2018).

Insect-fungal interactions are much more complex than vector-cargo relationships and have domino effects on host colonization (Schulthess et al., 2002; Ako et al., 2003; Piesik et al., 2011; Döll et al., 2013; Drakulic et al., 2015; Drakulic et al., 2016). For example, AF is known to be toxic to CEW based on in vitro studies (Zeng et al., 2006). Recent work on Drosophila further suggests that aflatoxigenic A. flavus strains may have greater fitness than non-aflatoxigenic strains in the presence of insects (Drott et al., 2017). However, there has been no study on the implications of these observations in the context of $\mathrm{AF}$ production in crop hosts. It was fortuitous that we observed a preferential CEW infestation and increased FUM contamination in A. flavus resistant maize lines in two unrelated field trials. These observations were robust and derived from two different sets of resistant and susceptible maize lines (details in the Materials and Methods section). Since it is relevant to mycotoxin mitigation, we pursued to unravel the factors underlying this novel host-pathogeninsect interaction. Late flowering might have facilitated enhanced oviposition by $\mathrm{H}$. zea in the resistant maize lines, but our analysis suggests that the toxicity of AF to CEW is a more compelling reason for the observed prevalence of ear damage in the low $\mathrm{AF}$ accumulating genotypes.

\section{MATERIALS AND METHODS}

\section{Maize Field Trials Related to the Study}

Field experiments were carried out at the Agricultural Research Station, LSU AgCenter, Baton Rouge. The four maize genotypes used in both trials are non-transgenic and non-commercial lines. The first or "hybrid" study used two hybrids, GA209 $\times$ T173 (susceptible to AF accumulation) and Mp313E $\times$ Mp717 (resistant to AF accumulation) that were developed at the USDA-ARS Corn Host Plant Resistance Research Unit, Mississippi (Williams and Windham, 2009). Toxigenic A. flavus strain, CA14 (obtained from the USDA Agricultural Research Service Culture Collection, Northern Regional Research Laboratory, Peoria, IL, USA) was used in the study. The strain has whole genome sequence information and needed mutant resources (Chang et al., 2019). The second or "inbred" 
study was done with two popular inbreds B73 (susceptible to AF accumulation, (Campbell and White, 1995) and CML322 (resistant to AF accumulation, (Betrán et al., 2002). Tox4, an isolate from local maize fields (Chalivendra et al., 2018), was used in the study because it is produces high AF levels and serves as a good model strain to study microbiome changes, which is the planned objective of the study.

All four lines were planted in 4-row plots in the middle of April, 2018. To keep the insect pressure low, Besiege, a broadspectrum foliar insecticide with chlorantraniliprole and $\lambda$ cyhalothrin as active ingredients, was sprayed at $\sim \mathrm{V} 9$ and $\mathrm{R} 1$ growth stages. Three days after the second insecticide application, plants were inoculated with conidial suspensions of A. flavus strains by silk canal injections, as described before (Zummo and Scott, 1992). Plants were maintained with standard agronomic practices of fertilizer and herbicide applications and received irrigations during extended dry periods.

\section{Weather Data}

The 2018 cropping season in the US was unusual in its weather pattern. Daily high and low temperatures and rainfall data were downloaded from https://www.wunderground.com/history/ monthly/us/la/baton-rouge/KBTR/ for April to July months of maize cropping season in 2017 and 2018 and are shown in Figure S1.

\section{Assessment of Earworm Damage and Mycotoxin Measurements}

One ear per plant from each genotype and treatment was harvested, resulting in 70-80 ears in inoculated plants and double the number from uninoculated plants. Ears in each lot were separated by the presence or absence of CEW infestation to monitor the effect of insect damage on mycotoxin levels. Only ears with visible internal damage (i.e., nibbled seed and cut silks, larval feeding tracks with frass; sometimes with dead or live CEW larvae) were considered as infested. No distinct spatial or other pattern of infestation was observed in our plots (also see Ni et al., 2011), except that a majority of resistant inbred or hybrid plants were infested, while only a few ears from susceptible lines showed damage by the earworm. At least three ears were used per replicate and each category had 3-5 replicates. Given the low frequency of CEW-damaged ears in B73 and GA209 × T173, all ears in each category were used for AF analysis to have robust $\mathrm{AF}$ data. When the seed meal exceeded more than $100 \mathrm{~g}$ (in uninoculated controls), we took more than one sample to minimize sampling error.

AF from seed meal was extracted and measured as before (Chalivendra et al., 2018) using modified high performance liquid chromatography (HPLC) conditions. The equipment included Waters e2695 HPLC (Waters Corp., Milford, MA, United States) fitted with a Nova-Pak C18 column, a photochemical reactor (Aura Industries Inc., New York, United States) and a Waters 2475 FLR Detector (Waters Corp.). The signal was detected by excitation at $365 \mathrm{~nm}$ and emission at $440 \mathrm{~nm}$. Aqueous methanol (37.5\%) was used as the mobile phase.
$\mathrm{FB}_{1}, \mathrm{FB}_{2}$, and $\mathrm{FB}_{3}$ in the same maize seed meal samples were analyzed by liquid chromatography-mass spectrometry (LCMS) using an adaptation of a previously published method for mycotoxin analysis (Plattner, 1999). Briefly, maize samples were ground with a laboratory mill. Portions $(5 \mathrm{~g})$ of the seed meal were extracted with $25 \mathrm{~mL} \mathrm{1:1} \mathrm{acetonitrile/water} \mathrm{for} 2 \mathrm{~h}$ on a Model G2 Gyrotory Shaker (New Brunswick Scientific, Edison, NJ, USA). Extracts were filtered with a Whatman $125 \mathrm{~mm} 2 \mathrm{~V}$ paper filter (GE Healthcare Bio-Sciences, Pittsburgh, PA, USA). A total of $10 \mu \mathrm{L}$ of extract was applied to a Kinetex (Phenomenex, Torrance, CA, USA) C18 column (50 mm length, $2.1 \mathrm{~mm}$ diameter). Chromatography was conducted utilizing a Thermo Dionex Ultimate 3000 (Thermo Fisher, Waltham, MA, USA) ultrahigh-performance liquid chromatography (UPLC) system consisting of an autosampler coupled to a binary gradient pump. Elution of analyte was achieved with a $0.6 \mathrm{~mL} \mathrm{~min}^{-1}$ gradient flow of methanol and water $(0.3 \%$ acetic acid was added to the mobile phase). The solvent program used a 35-95\% gradient over $5 \mathrm{~min}$. Flow was directed to a Q Exactive (Thermo Fisher, Waltham, MA, USA) hybrid quadrupole-Orbitrap mass spectrometer equipped with an electrospray ionization source. The mass spectrometer was operated in full-scan mode over a range of 300 to $1,200 \mathrm{~m} / \mathrm{z}$. Operation of the LC-MS and quantification of the eluting fumonisins were performed utilizing Thermo Xcalibur software. Quantification of fumonisins was based upon intensity of protonated ions for $\mathrm{FB}_{1}(\mathrm{~m} / \mathrm{z} 722.3), \mathrm{FB}_{2}(\mathrm{~m} / \mathrm{z} 706.3)$ and $\mathrm{FB}_{3}(\mathrm{~m} / \mathrm{z} 706.3)$ compared to calibration standards of the toxins. The limit of quantification for the analytical method was determined to be $0.1 \mu \mathrm{g}$ per $\mathrm{g}$ for $\mathrm{FB}_{1}, \mathrm{FB}_{2}$, and $\mathrm{FB}_{3}$.

\section{Bioassays for Mycotoxin Toxicities to CEW}

The toxicities of AF and FUM to CEW larvae were tested in a pre-mixed meridic diet (WARD'S Stonefly Heliothis diet, Rochester, NY) by supplementing with $0,3,10,3060$, or 100 $\mu \mathrm{g} / \mathrm{g} \mathrm{FB}{ }_{1}$ (Cayman Chemical, MI) or $0,20,50,100,250$, or 500 $\mathrm{ng} / \mathrm{g}$ of $\mathrm{AFB}_{1}$ (Sigma Chemicals). The diet was prepared as per manufacturer's instructions. The $\mathrm{FB}_{1}$ stock, made in water, was diluted to the above rates before the dry diet was added and mixed thoroughly. AF was dissolved in methanol at a stock concentration of $2 \mathrm{mg} / \mathrm{mL}$ and diluted appropriately to provide the aforementioned concentrations. The highest concentration of methanol used $(0.08 \%$ by $\mathrm{w} / \mathrm{w})$ was incorporated into the control diet. The assay was done in a 128 well bioassay plate $(\mathrm{C}-\mathrm{D}$ International Inc., Pitman, NJ). A single CEW neonate from a laboratory $\mathrm{CEW}$ colony obtained from Benzon Research Inc. (Carlisle, PA) was added to each well with $1 \mathrm{~g}$ of diet using a camel hair brush (Kaur et al., 2019). At least 20 larvae were tested per treatment and the assay was repeated four times.

\section{Statistical Analysis of Data}

Insect damage and aflatoxin levels were compared by ANOVA and post-hoc analysis by Tukey's Honestly Significant Difference (HSD) test using R program (version 3.6.2) in RStudio. Student's t-test was used for comparison of specific pairs of data sets. 


\section{Safety}

$\mathrm{AF}$ and $\mathrm{FB}_{1}$, being highly toxic mycotoxins, were handled with care using a biohood, surgical gloves and nose as well as mouth masks. All residues and containers were decontaminated using bleach and by autoclaving.

\section{RESULTS}

\section{Corn Earworm Outbreak in 2018 Summer}

The unexpected observation that prompted the current work was made in two separate field experiments in 2018. The objective of hybrid study was to correlate the transcripts of A. flavus medusa A gene with the spatial distribution of the biofilm-like structure in maize seeds. Previous studies showed that A. flavus forms biofilmlike structure during maize seed colonization (Dolezal et al., 2013; Shu et al., 2014; Windham et al., 2018). The aim of the inbred study was to analyze microbiome changes in the susceptible and a resistant line in response to A. flavus colonization.

During the summer of 2018, daily profiles of rain fall and air temperature patterns were different from past years' average in Louisiana as well as many of the maize-growing states in US. The growing season was shorter (late April to early August) due to extended cold temperatures into the beginning of the planting season and relatively warmer and drier days during the early crop growth period (Figure S1). April 2018 was the coldest April month since 1997 based on US average temperatures (and for Iowa and Wisconsin, it was the coldest April since records began in 1895). In contrast, May 2018 was the hottest May on record, breaking the record set in May 1934 during the Dust Bowl (National Oceanic and Atmospheric Administration: https:// www.noaa.gov/). The unseasonal and steep warming, and the dry weather after protracted cold seems to have favored an explosion of CEW population as indicated by a heavy infestation of ears in both of our experimental plots. CEW incidence was also reported from maize fields in other states in southern (Porter and Bynum, 2018) as well as northern US (e.g., Handley, 2018). A similar buildup of CEW reported in Michigan in 2019 was also attributed to unusual weather pattern (Schuh and Springborn, 2019). In spite of two applications (before and after silking) of a strong broad-spectrum insecticide with fast knockdown as well as long-lasting residual effects, the insecticide seems to have failed to reach silks covered by the husks. Further, all ears were bagged immediately after inoculation/pollination, which concealed earworm damage until developing ears were sampled for analysis.

\section{CEW Infestation Was Significantly Greater in A. flavus Resistant Maize Lines}

During sampling of ears later in the season (July), we noticed that the two resistant lines, the hybrid Mp313E $\times$ Mp717 and the inbred CML322 showed greater infestation by CEW than the susceptible lines GA209 × T173 and B73 (Figure 1, left panels). The infestation was $<10 \%$ in susceptible lines and it ranged from 22 to $68 \%$ in the resistant lines. The maize lines used in the two field trials have been extensively validated in the field and are often used as checks for evaluating new genotypes and in mapping resistance loci (e.g., Mideros et al., 2012; Guo et al., 2017). Despite our concerns that the distinctive patterns of CEW infestation might potentially interfere with the genetic response of maize lines to A. flavus, AF measurements showed that the genotype responses were robust in spite of CEW infestation. As described in the Materials and Methods section, we harvested and utilized all ears in the plots to obtain robust AF data. The insect infestation was 8-fold greater in CML322 than observed in B73 ears in the mock-inoculated set. Inoculation with the highly toxigenic Tox4 strain resulted in a significant $(\mathrm{p}<0.01)$ and nearly 4 -fold decrease in the infestation of CML322, but still 2-fold greater than infestation in B73. This is inversely correlated with $>3$-fold increase in seed AF content in Tox4- inoculated CML322 ears. As expected from its susceptibility to A. flavus colonization, B73 seeds accumulated $>100 \mathrm{ppb}$ of AF even in mock-inoculated (Control) ears and $>500 \mathrm{ppb}$ in Tox4-inoculated ears. These AF levels are $>12-19$ fold higher than those measured in CML322 seeds (Figure 1B, right panel). CEW infestation was also greater in the resistant hybrid $(\mathrm{Mp} 313 \mathrm{E} \times \mathrm{Mp} 717)$ than in the susceptible hybrid by $>30$-fold in the control set and by 7 -fold in the inoculated set (Figure 1A, left panel). Infestation was inversely correlated with seed AF levels in hybrids as well. The susceptible hybrid (GA209 $\times$ T173) had $100 \mathrm{ppb}$ of AF in uninoculated control seeds and $>400 \mathrm{ppb}$ in the inoculated set (i.e., 3 and 24-fold greater than in the resistant hybrid). Unlike the resistant inbred CML322, the resistant hybrid showed no difference in either AF content or CEW infestation between the control and CA14-inoculated ears. Analysis of variance (ANOVA) confirmed that only the host genotype (i.e., resistance to $A$. flavus) affected infestation highly significantly (>99.99\% confidence level) and inoculation-induced differences were not statistically different (Table S1).

\section{CEW Infestation Is Negatively Correlated With Seed AF Content}

As can be expected from the above data (Figure 1), ANOVA of seed AF content across the two experiments (Table S2) revealed that the host genotype and inoculation with toxigenic A. flavus strains showed highly significant direct as well as interaction effects on seed AF content. Infestation was also significantly related to AF content, although its interaction effect with genotype on AF was not significant. Both the resistant genotypes (CML322 and Mp313E $\times$ Mp717) manifested robust resistance to A. flavus and accumulated less than $30 \mathrm{ppb}$ of AF in the seed either in the control (via colonization of native A. flavus strains) or the inoculated set. Conversely, the susceptible inbred and hybrid accumulated 100 and $500 \mathrm{ppb}$ in control and inoculated sets, respectively. AF content is inversely correlated with CEW infestation pattern in each of the four maize genotypes. This relationship becomes clear when the data is combined for control and inoculated sets in each genotype (Figure 2) or when all data is combined (Figure S2). It is of interest to note that the uninfected controls from both resistant lines showed a numerical but statistically insignificant increase in $\mathrm{AF}$ in $\mathrm{CEW}$-infested ears. AF was scarcely detectable in the 

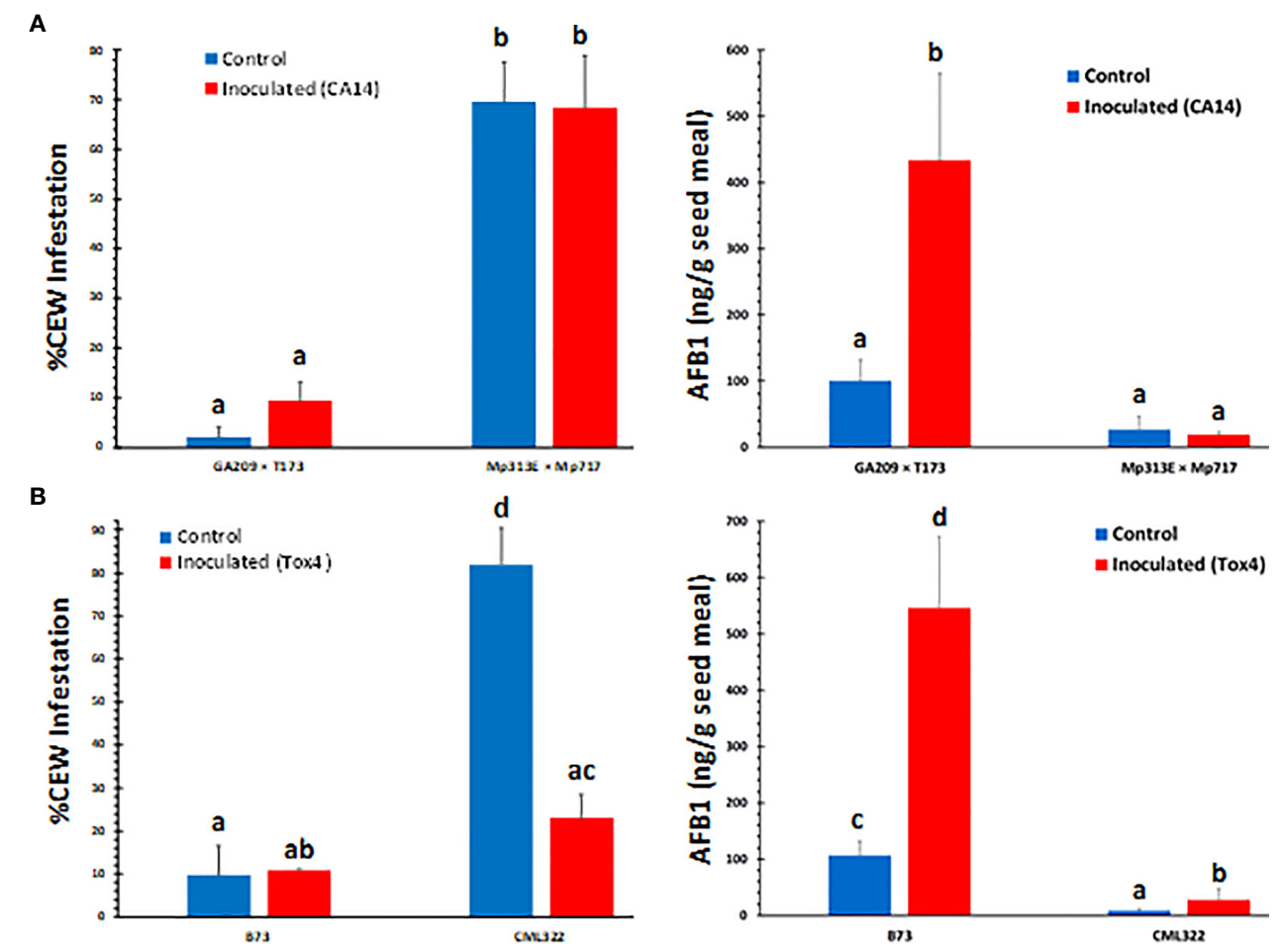

FIGURE 1 | Rate of corn earworm infestation (left panels) and seed AF content (right panels) in maize lines. (A) Data is from hybrid plots. Infestation was significantly dependent on the host genotype with very little difference between control (mock-inoculated) and CA14-inoculated set. (B) Data shown is from inbreds. Values shown are average + SE. Significant differences (P value <0.05) between each data set were tested using an ANOVA (Table S1) followed by Tukey's multiplecomparisons post hoc test in R (version 3.6.2). Means are significantly different if marked by a different letter.

uninfested and uninoculated controls (a mean value of $6 \mathrm{ppb}$ in Mp313E $\times$ Mp717 and $<1 \mathrm{ppb}$ in CML322) but increased by 5 and 14-fold in infested ears of resistant hybrid and inbred respectively. This suggested that resistance to AF contamination might have been compromised to some extent in seeds heavily damaged by CEW.

\section{Kernel Fumonisin Content Was Enhanced in CEW-Infested Ears}

Fusarium verticillioides is among the most common mycotoxigenic fungi colonizing field-grown maize. We observed symptoms of $F$. verticillioides colonization (e.g., star-burst pattern on seeds) in our samples. We isolated the fungus from seeds with visual symptoms using Fusarium-selective Malachite Green Agar 2.5 medium (Alborch et al., 2010) and confirmed the species identity by genomic PCR using $F$. verticillioidesspecific primers (Baird et al., 2008). FUM content was analyzed in the same seed samples used for AF determination (Figure 3A) and compared between uninfested and CEWinfested samples (Figure 3B).

Both maize hybrids used in this study have been previously shown to be resistant to FUM accumulation. The A. flavus resistant hybrid, $\mathrm{Mp} 313 \mathrm{E} \times \mathrm{Mp} 717$ was shown to be more robustly resistant than GA209 $\times$ T173 in multiple studies (Williams, 2006; Henry et al., 2009; Williams and Windham, 2009). In the current study, however, the Mp313E × Mp717 accumulated $>7$-fold greater concentration of FUM in its seeds than GA209 $\times$ T173 (Figure 3A). Although CML322 accumulated a considerable amount of FUM, it was $>4$-fold less than that in B73, which is known to be among the most susceptible inbreds to Fusarium ear rot and FUM accumulation (Morales et al., 2019). However, when the data was parsed based on CEW infestation (only in sets where both clean and infested ears were available), infested ears showed $>5$-fold more FUM than uninfested ears (Figure 3B). The differences were not significant probably due to the high variability in the colonization by native strains (the lowest $p$-value was 0.052 for CML322; also see Figure S2). These data indicated that CEW may vector Fusarium spp. that produce FUM during its infestation, as often reported in the literature (Munkvold et al., 2019).

\section{Differential Toxicity of AF Versus $\mathrm{FB}_{1}$ to CEW}

The preferential infestation of A. flavus resistant lines by CEW, the negative correlation between $\mathrm{AF}$ and CEW infestation levels, and a greater FUM levels in infested ears, suggested that AF may be more toxic to H. zea than FUM. We tested this hypothesis by feeding experiments where $\mathrm{CEW}$ neonates were reared on artificial diet containing graded levels of $\mathrm{AF}$ or $\mathrm{FB}_{1}$. Results shown in Figure $\mathbf{S 3}$ and Figures $\mathbf{4 A}, \mathrm{B}$ clearly demonstrate that the pest is more susceptible to $\mathrm{AF}$ than to $\mathrm{FB}_{1}$. As reported 

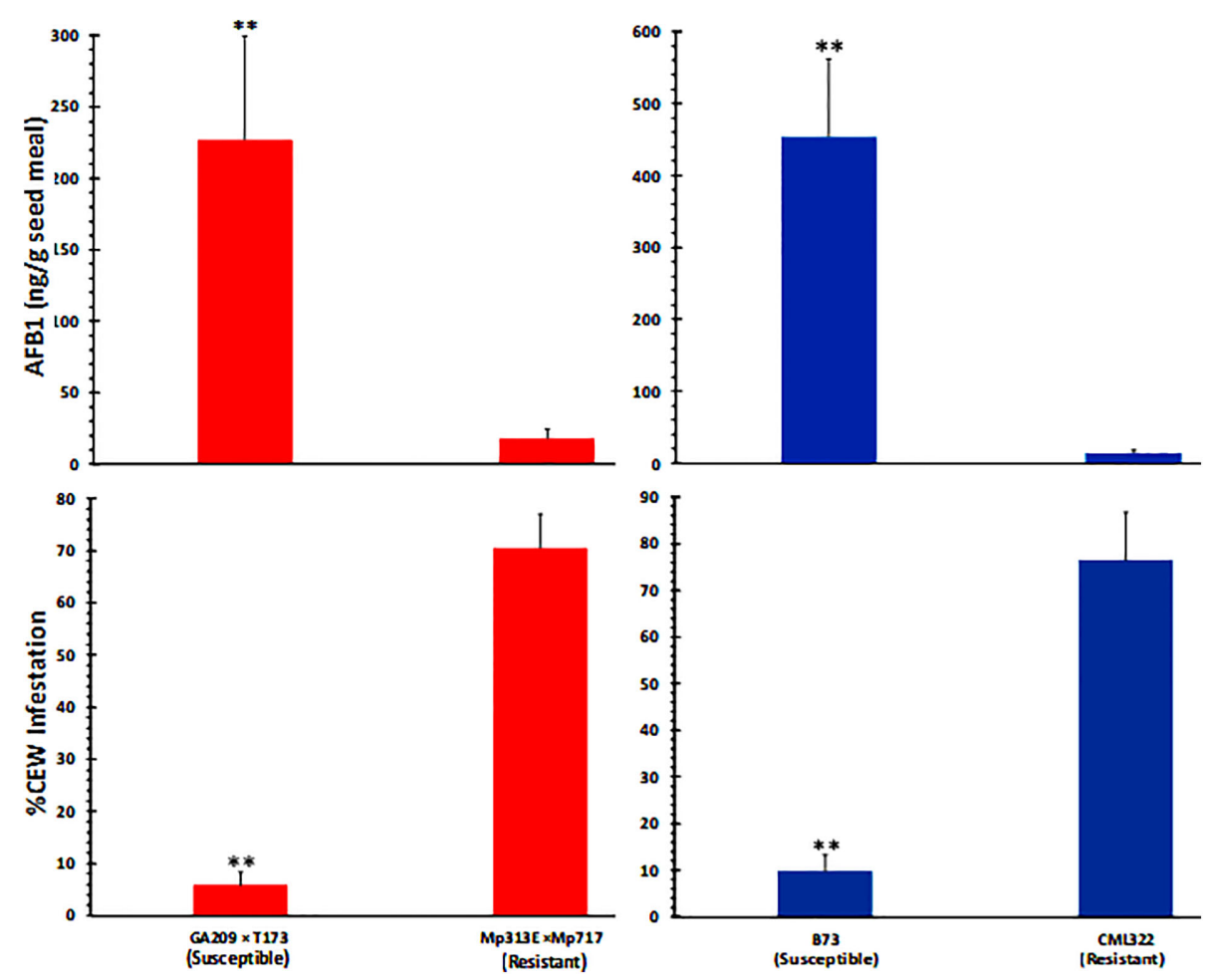

FIGURE 2 | CEW damage is negatively correlated with seed AF content in maize 20 lines. The infestation and AF data from control and infected ears is combined in each genotype. Significant differences ( $P$ value $<0.05$ ) between each data set were tested using an ANOVA followed by Tukey's multiple-comparisons post hoc test in R. Average (+SE) infestation and AF values between A. flavus susceptible and resistant lines are highly significant (denoted by ${ }^{\star *} ; \mathrm{p}<0.01$ ).

previously (Zeng et al., 2006), AF retarded CEW larval growth even at the lowest concentration tested, although the effect was not significant (Figure 4B) and was lethal above $200 \mathrm{ppb}$ (Figure S3). On the other hand, $\mathrm{FB}_{1}$ had little impact on CEW larval growth at all concentrations tested (Figure S3 and Figure 4A). In fact, at lower concentrations (below 30 ppm; Figure S3) the toxin seems to marginally enhance the growth of the larvae (the effect was consistent although there was variability among the bioassays). These results support our proposal that the enhanced infestation of $A$. flavus resistant maize lines by $H$. zea may be due to very low levels of AF that are not inhibitory to larval growth.

\section{Delayed Flowering in A. flavus Resistant Maize Lines}

Tassel and ear development was delayed in the resistant inbred CML322 by 3 weeks relative to B73 and by $4-5$ weeks in the resistant hybrid, Mp313E $\times$ Mp717 compared to GA209 × T173, although all four lines were planted together. CML322 is a tropical inbred and manifests delayed flowering under long days, i.e., $\geq 13 \mathrm{~h}$ photoperiod (Hung et al., 2012). The parents of the resistant hybrid $(\mathrm{Mp} 313 \mathrm{E} \times \mathrm{Mp} 717)$, derived from the tropical maize race Tuxpeño (Scott and Zummo, 1990; Williams and Windham, 2006), are also known to flower late. This is true for most maize lines that are resistant to A. flavus. Attempts to segregate the two traits, so far, have met with limited success (Henry, 2013). The availability of green silks may be an important factor for an increased $H$. zea infestation of often observed in the late flowering genotypes. However, in an adjacent plot where B73 was planted two weeks later (unrelated to the current study), silk emergence coincided with that of CML322 plants used in the present study. Nonetheless, B73 ears showed low levels of CEW infestation correlating with highly elevated levels of seed AF (400 ppb in controls and 800 $\mathrm{ppb}$ in inoculated plants) in this plot as well. These observations suggest that high seed AF levels suppress CEW infestation due to its toxicity, even if provision of green silks can promote CEW egg-laying.

\section{DISCUSSION}

The precise environmental factor that favored a CEW outbreak in 2018 is not clear. Unseasonal dry and warm weather is considered to support CEW population buildup in soybean but negatively affect infestation of drought-stressed maize (Herbert et al., 2003). For example, CEW damage was more severe in 2006 than in 2007 in the same maize field (Ni et al., 2011), although 2007 set the record as the then driest year in Georgia's history (www.drought.gov/drought/states/georgia). Seasonal outbreaks of CEW population have been reported in the past but detailed correlative analysis between seasonal weather and CEW 

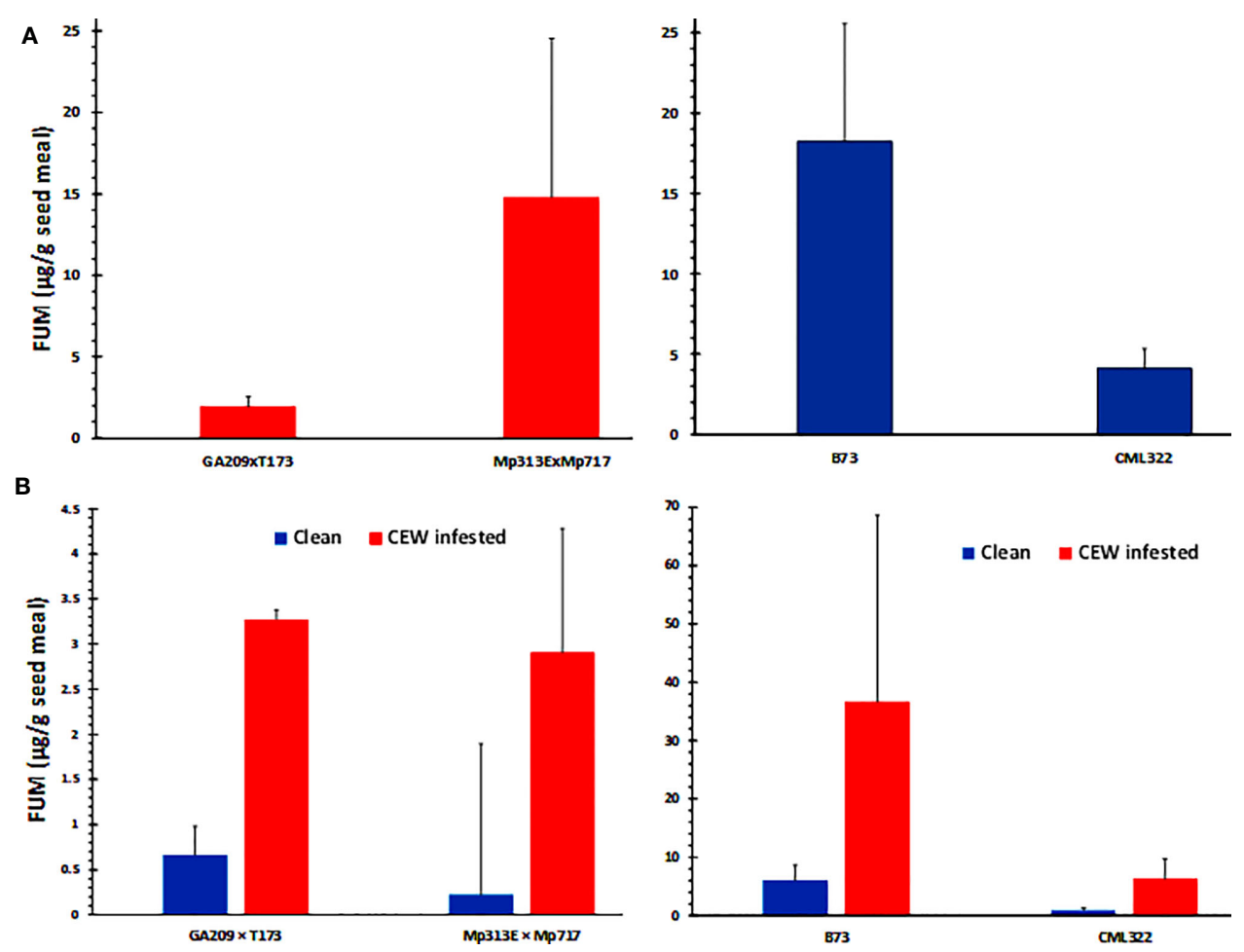

FIGURE 3 | FUM contamination by native Fusarium strains. (A) Seed fumonisin content in the four maize lines. (B) Seed FUM content parsed by uninfested (clean) versus CEW infested ears in each genotype. The values are averages + SE in each genotype and were not significantly different at $95 \%$ confidence level.

A

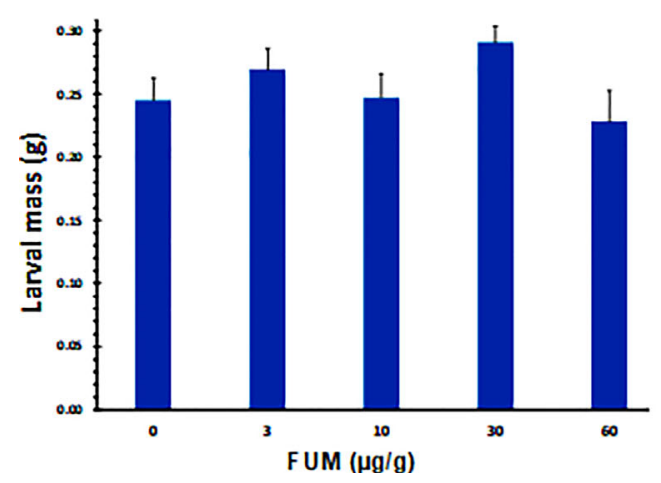

B

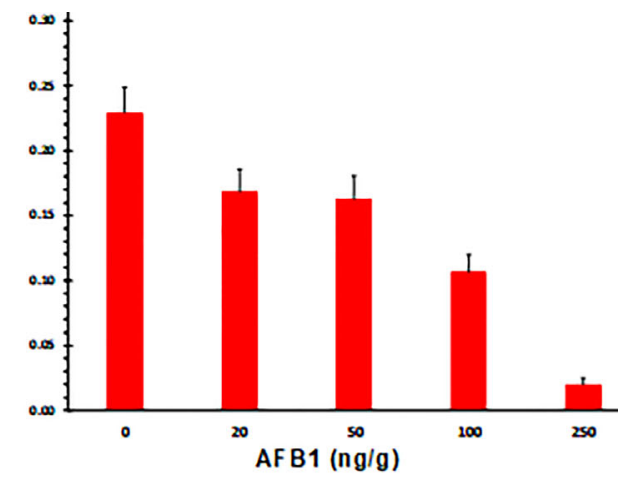

FIGURE 4 | Effects of aflatoxin $B_{1}$ and fumonisin $B_{1}$ on the growth of $H$. zea larvae, as measured by the body mass. Graded doses of $F B_{1}$ and $A F(\mathbf{A}, \mathbf{B}$ respectively) were tested on CEW growth and mortality by incorporating them into an artificial insect diet. Larvae were grown in a 128 well bioassay plate for $10 \mathrm{~d}$. Each well had $1 \mathrm{~g}$ of feed and a single neonate at the start of the assay. A representative assay from 4 replicated experiments is shown (Figure S3). At the end of the bioassay, larvae were removed from the well killed by chloroform vapors and weighed. Values are averages $+\mathrm{SE}$ of $\geq 16$ larvae/treatment except at $250 \mathrm{ppb}$ of AF, where mortality was $30 \%$ or greater (dead and dried larvae were seen stuck to the bottom of the well). The values marked with the same letter are not statistically significant. $\mathrm{FB}_{1}$ had no significant effect on larval growth at concentrations tested.

outbreaks is lacking. In a period of 25 years, severe crop damage by CEW was recorded during 9 of them in the state of Arkansas and no comparison to prevailing weather factors was made (reviewed in Dicke, 1939). There has also been no study where CEW infestation patterns have been compared in maize genotypes with varying resistance to A. flavus or AF accumulation, although toxicity of AF to CEW has been known for more than a decade (Zeng et al., 2006). Ni et al. (2011) compared spatial patterns of natural infestation of four ear-feeding insects including CEW with AF contamination by 
native $A$. flavus strains in a commercial maize hybrid. In the first year of the study, CEW infestation was extensive (95\% of sampled ears) and in the second year, it was $41 \%$. Although the low AF content observed in both years ( $>80 \%$ of ears had $\leq 30$ $\mathrm{ppb}$ and only $\leq 4 \%$ ears had $\leq 100 \mathrm{ppb}$ ) makes it difficult to quantify the relationship between AF contamination and insect infestation, it indicated a negative association between CEW damage and seed AF content. The maize genotypes in our study have proven resistance or susceptibility to A. flavus. Further, high AF contamination (100 ppb) even in uninoculated susceptible lines has allowed to make robust comparisons.

The premise for this study is a novel and robust observation in that two unrelated maize lines (Tuxpeño germplasm versus $\mathrm{CML}$ ) with proven resistance to $A$. flavus were heavily infested by CEW. Conversely, the two A. flavus susceptible lines (stiff-stalk inbred B73 and non-stiff stalk hybrid GA209 $\times$ T173) were spared by the pest. Although late flowering maize is known to be susceptible to CEW infestation by providing green silks, availability of silks alone could not fully explain our observations. Late flowering is more often a problem in the northeastern US where it coincides with CEW migration from southern states. Furthermore, late planted B73 in an adjacent plot had delayed silk emergence but showed no greater CEW infestation than the early planted set. The other and more likely explanation is that the susceptible lines had very high levels of AF that were toxic to CEW. Even mock-inoculated controls had 100 ng of AF per gram of seed meal prepared from entire ears that included both moldy and non-moldy seeds. This inference is supported by our feeding experiments (Figure S3 and Figure 4B) as well as previous work (Zeng et al., 2006). Zeng et al. (2006) showed that AF at $200 \mathrm{ppb}$ strongly inhibited the growth and development of first instar larvae, leading to $>50 \%$ larval death after $9 \mathrm{~d}$ and $100 \%$ death after $15 \mathrm{~d}$ of feeding. Even lower concentrations (1-20 ppb; FDA-regulated levels) affected larval development, delayed pupation rate and led to $>40 \%$ mortality when the exposure was longer than 7 d (Zeng et al., 2006). Although concentrations below $20 \mathrm{ppb}$ were not tested in our study, we observed a steady decline in larval mass as AF concentration increased resulting in $\geq 30 \%$ mortality at or above $250 \mathrm{ppb}$ during 10-15 d exposure (Figure S3 and Figure 4B). We did not continue our observations beyond the larval stage to assess long-term developmental effects (e.g., pupation or emergence of adults). An apparent exception to the correlation between low AF and high CEW infestation was a significant decrease in CEW infestation observed in TOX4inoculated ears compared to uninoculated ears in the A. flavus resistant inbred CML322, although average AF levels did not exceed $30 \mathrm{ppb}$. Given the highly variable distribution of AF in individual kernels of a maize ear (e.g., Lee et al., 1980), it is possible that the AF content particularly in damaged kernels at the ear tip was much greater than the average for the entire ear and high enough to be toxic to CEW. Furthermore, CEW may be sensitive also to other anti-insectan compounds that can be made by A. flavus (Cary et al., 2018) and act additively or synergistically with AF (e.g., Kojic acid; Dowd, 1988). Future experiments would involve late-maturing lines with $A$. flavus susceptibility and early maturing lines with $A$. flavus resistance to clarify and quantify the effects of flowering time and AF content on CEW infestation.

It is not surprising that $\mathrm{AF}$ is toxic to insects, not merely to mammals. A. flavus is predominantly a soil-living saprophyte, feeding on decaying organic matter, including dead insects. It is also an opportunistic pathogen and can colonize a wide variety of insects, e.g., moths, silkworms, bees, grasshoppers, houseflies, fruit flies and mealy bugs among others (St. Leger et al., 2000; Gupta and Gopal, 2002) and AF production may be an adaptive mechanism against fungivory (Drott et al., 2017). A. flavus is known to survive ingestion by mycophagous insects. Among three Aspergillus species tested, A. flavus conidia phagocytized by insect hemocytes were still able to germinate (St. Leger et al., 2000). A. flavus may also proliferate in the hindgut of CEW (Abel et al., 2002). Based on feeding studies in Drosophila, AF production is proposed to confer a fitness advantage to $A$. flavus when interacting with insects (Drott et al., 2017). In spite of being a polyphagous pest with a remarkable capacity to metabolize a wide array of plant compounds, CEW has limited tolerance to $\mathrm{AF}$ and poor ability to metabolize this mycotoxin (Dowd, 1988; Zeng et al., 2006). Beside AF, the fungus is known to make several anti-insectan compounds (TePaske et al., 1992; Cary et al., 2018). Other insect pests that are more tolerant may vector A. flavus (Zeng et al., 2006; Opoku et al., 2019; Reviewed in Munkvold et al., 2019). Based on spatial correlation analysis, $\mathrm{Ni}$ et al. (2011) reported that AF content was correlated to the frequency of weevils and stink bug-affected kernels, but not with CEW damage.

Our work also showed that FUM is not toxic to H. zea (Figure S3 and Figure 4A). This may have allowed CEW to vector $F$. verticillioides and other FUM-contaminating fungi, as indicated by an increased seed FUM content in infested ears (Figure 3). CEW damage is also frequently associated with the colonization by another mycotoxigenic fungus, Stenocarpella maydis, which causes diplodia ear rot (Munkvold and White, 2016). In animal model systems, $\mathrm{FB}_{1}$ at 25-50 $\mu \mathrm{M}$ (i.e., 18-36 ppm) has been shown to inhibit ceramide synthases and lead to the accumulation of toxigenic/carcinogenic sphinganine and related compounds (Riley et al., 2001; Riley and Merrill, 2019). Conversely, $\mathrm{FB}_{1}$ was not found to be toxic even at $450 \mathrm{ppm}$ to yellow mealworm larvae when included in the diet or injected into larva (Abado-Becognee et al., 1998). Recently, the brown marmorated stink bug (Halyomorpha halys) was shown to enhance $F$. verticillioides infection and FUM contamination in field corn (Opoku et al., 2019). Among other secondary metabolites produced by $F$. verticillioides, fusaric acid is only a weak antisectan compound (Dowd, 1988). The lack of secondary metabolites with potent insecticidal properties in the biosynthetic repertoire of $F$. verticillioides could be one of the reasons for its frequently observed transmission via insect infestation (e.g., Smeltzer, 1959; Dowd, 2000; Alma et al., 2005; Mesterházy et al., 2012; Madege et al., 2018) and a critical link between insect damage and Fusarium ear rot (Munkvold et al., 2019). Successful mitigation of mycotoxins requires control of multiple pests, including CEW (Abbas et al., 2013; Bowers et al., 
2014; Porter and Bynum, 2018). Bt-maize has been highly successful in crop protection from important pests, including CEW. However, global warming has been shown to enhance the risks of extensive Bt-adaptation as well as overwintering of CEW in the northern US (Venugopal and Dively, 2017) and could exacerbate the mycotoxin problem.

Although this study was pursued to explain a serendipitous observation, it has important implications in mycotoxin control. AF and FUM are ubiquitous and unpredictable contaminants of commodities, particularly maize. Our study clarifies a component of this unpredictability. The late flowering trait of A. flavus resistant lines (owing to their tropical origin) is known to delay harvest, potentially leading to frost damage and/or high grain moisture. Our current work shows that delayed flowering when coupled with unseasonal weather and low AF accumulation can exacerbate CEW infestation, which in turn can lead to contamination by other mycotoxins, such as fumonisins (Munkvold and White, 2016).

In contrast to a mutual antagonism reported previously between A. flavus and F. verticillioides (Zummo and Scott, 1992; also see Figure S4), we observed high levels of AF and FUM cocontaminating our samples. B73, in particular with its high susceptibility to both mycotoxigenic fungi, had very high levels of both AF and FUM in many of its seed samples. Although CEW damage was very low in this inbred (Figures 1B and 2), FUM levels were exacerbated in infested ears (Figure 3B). There is some evidence for an additive or even synergistic effect on carcinogenicity from co-exposure to AF and FUM (World Health Organization, 2018). Based on biomarker studies and food analyses, the cooccurrence of these two mycotoxins has been widely documented in developing countries (Shirima et al., 2013; Biomin, 2019). It is important to examine the underlying factors as well as effects of mycotoxin co-contamination both by researchers and regulatory agencies to mitigate its impact on food safety (Lopez-garcia, 1998). As demonstrated by our study, a host genotype even with demonstrable resistance can become vulnerable due to seasonal variation in flowering time or an outbreak of chewing insects. Further, incorporation of resistance to a single mycotoxin accumulation and not pairing it with insect resistance may not adequately ensure food safety.

\section{REFERENCES}

Abado-Becognee, K., Fleurat-Lessard, F., Creppy, E. E., and Melcion, D. (1998). Effects of fumonisin B1 on growth and metabolism of larvae of the yellow mealworm, Tenebrio molitor. Entomol. Experiment. Applicata 86, 135-143. doi: 10.1046/j.1570-7458.1998.00274.x

Abbas, H. K., Zablotowicz, R. M., Weaver, M. A., Shier, W. T., Bruns, H. A., Bellaloui, N., et al. (2013). Implications of Bt Traits on Mycotoxin Contamination in Maize: Overview and Recent Experimental Results in Southern United States. J. Agric. Food Chem. 61, 11759-11770. doi: 10.1021/ jf400754g

Abel, C. A., Abbas, H. K., Zablotowickz, R., Pollan, M., and Dixon, K. (2002). "The association between corn earworm damage and aflatoxin production in preharvest maize grain" in Proceedings of the 2nd Fungal Genomics, 3rd Fumonism Elimination and 15th Aflatoxin Elimination Workshop, Eds. J. F. Robens and R. L. Brown (Beltsville, MD: U.S.D.A., Agricultural Research Service), 113-114.

Ako, M., Schulthess, F., Gumedzoe, M. Y. D., and Cardwell, K. F. (2003). The effect of Fusarium verticillioides on oviposition behaviour and bionomics of

\section{DATA AVAILABILITY STATEMENT}

The raw data supporting the conclusions of this article will be made available by the authors, without undue reservation.

\section{AUTHOR CONTRIBUTIONS}

SC planned the study. SC, MB, and FH carried out the experiments. WW, JH contributed resources. SC assembled and analyzed the data, and wrote the manuscript. All authors contributed to the article and approved the submitted version.

\section{FUNDING}

SC thanks the National Corn Growers Association for the funding support through their AMCOE program.

\section{ACKNOWLEDGMENTS}

Excellent field and laboratory assistance by Mr. Anthony Nguyen is gratefully acknowledged. Dr. Z-Y Chen is thanked for his help in aflatoxin measurements. We thank Dr. Geromy Moore (USDA-ARS, Southern Regional Research Center, New Orleans) for carefully going through the manuscript and Dr. Umesh Reddy, West Virginia State University for the help in formatting the figures. This manuscript has been released as a pre-print at BioRxiv, Chalivendra et al., 2020 (www.biorxiv.org/ content/10.1101/2020.02.03.933309v1).

\section{SUPPLEMENTARY MATERIAL}

The Supplementary Material for this article can be found online at: https://www.frontiersin.org/articles/10.3389/fpls.2020. 565323/full\#supplementary-material

lepidopteran and coleopteran pests attacking the stem and cobs of maize in West Africa. Entomol. Experiment. Applicata 106, 201-210. doi: 10.1046/ j.1570-7458.2003.00026.x

Alborch, L., Bragulat, M. R., and Cabañes, F. J. (2010). Comparison of two selective culture media for the detection of Fusarium infection in conventional and transgenic maize kernels. Lett. Appl. Microbiol. 50, 270-275. doi: 10.1111/ j.1472-765X.2009.02787.x

Alma, A., Lessio, F., Reyneri, A., and Blandino, M. (2005). Relationships between Ostrinia nubilalis (Lepidoptera: Crambidae) feeding activity, crop technique and mycotoxin contamination of corn kernel in northwestern Italy. Int. J. Pest Manage. 51, 165-173. doi: 10.1080/09670870500179698

Baird, R., Abbas, H. K., Windham, G., Williams, P., Baird, S., Ma, P., et al. (2008). Identification of select fumonisin forming Fusarium species using PCR applications of the polyketide synthase gene and its relationship to fumonisin production in vitro. Int. J. Mol. Sci. 9, 554-570. doi: 10.3390/ ijms 9040554

Betrán, F. J., Isakeit, T., and Odvody, G. (2002). Accumulation accumulation of white and yellow maize inbreds in diallel crosses. Crop Sci. 42, 1894-1901. doi: $10.2135 /$ cropsci2002.1894 
Biomin (2015). World Mycotoxin Survey. www2.biomin.net/fr/blog-posts/2015biomin-mycotoxin-survey-out-now/

Biomin (2019). World Mycotoxin Survey, Quarter 1. https://www2.biomin.net/pt/ artigos/biomin-mycotoxin-survey-q1-2019-results/

Blacutt, A. A., Gold, S. E., Voss, K. A., Gao, M., and Glenn, A. E. (2018). Fusarium verticillioides: advancements in understanding the toxicity, virulence, and niche adaptations of a model mycotoxigenic pathogen of maize. Phytopathology 108, 312-326. doi: 10.1094/PHYTO-06-17-0203-RVW

Bowers, E., Hellmich, R., and Munkvold, G. (2014). Comparison of Fumonisin Contamination Using HPLC and ELISA Methods in Bt and Near-Isogenic Maize Hybrids Infested with European Corn Borer or Western Bean Cutworm. J. Agric. Food Chem. 62, 6463-6472. doi: 10.1021/jf5011897

Boyd, M. L., and Bailey, W. C. (2001). European Corn Borer: A Multiple-Crop Pest in Missouri. Bulletin. G7113. https://extension2.missouri.edu/g7113.

P. P. Bynum (Ed.) (2018). Texas Panhandle: Bt Corn - What's Up With All the Corn Earworms? (AgriLife Extension, Texas A\&M University, College Station) Available at: https://focusonagriculture.blogspot.com/2018/07/whats-up-withall-corn-earworms-in-bt.html.

Campbell, K. W., and White, D. G. (1995). Evaluation of corn genotypes for resistance to Aspergillus ear rot. kernel infection and aflatoxin production. Plant Dis. 79, 1039-1045. doi: 10.1094/PD-79-1039

Cao, A., Santiago, R., Ramos, A. J., Souto, X. C., Aguín, O., Malvar, R. A., et al. (2014). Critical environmental and genotypic factors for Fusarium verticillioides infection, fungal growth and fumonisin contamination in maize grown in northwestern Spain. Int. J. Food Microbiol. 177, 63-71. doi: 10.1016/j.ijfoodmicro.2014.02.004

Capinera, J. L. (2004). Encyclopedia of Entomology (New York: Kluwer-Academic).

Cappelle, K. (2018). Fumonisin B1 in Bt and non-Bt maize: A meta-analysis (Arnes: Iowa State University Graduate Theses and Dissertations), 16324. doi: 10.31274/etd-180810-5954

Cary, J. W., Gilbert, M. K., Lebar, M. D., Majumdar, R., and Calvo, A. M. (2018). Aspergillus flavus secondary metabolites: more than just aflatoxins. Food Saf. 6 , 7-32. doi: 10.14252/foodsafetyfscj.2017024

Chalivendra, S., DeRobertis, C., Reyes Pineda, J., Ham, J. H., and Damann, K. (2018). Rice phyllosphere Bacillus species and their secreted metabolites suppress Aspergillus flavus growth and aflatoxin production in vitro and in maize seeds. Toxins 10, 159. doi: 10.3390/toxins10040159

Chalivendra, S. C., Busman, M., Huang, F., Willams, W. P., and Ham, J. (2020). Low aflatoxin levels and flowering delay in Aspergillus flavus-resistant maize lines are correlated with increased corn earworm damage and enhanced seed fumonisin. (www.biorxiv.org/content/10.1101/2020.02.03.933309v1). doi: 10.1101/2020.02.03.933309

Chang, P.-K., Scharfenstein, L. L., Mack, B., and Hua, S. S. T. (2019). Genome Sequence of an Aspergillus flavus CA14 Strain That Is Widely Used in Gene Function Studies. Microbiol. Res. Announcements 8, e00837-e00819. doi: 10.1128/MRA.00837-19

Cotty, P. J., and Jaime-Garcia, R. (2007). Influences of climate on aflatoxin producing fungi and aflatoxin contamination. Int. J. Food Microbiol. 119, 109-115. doi: 10.1016/j.ijfoodmicro.2007.07.060

Dicke, F. F. (1939). Seasonal abundance of the corn earworm. J. Agric. Res. 59, 237-258.

Dively, G. P., Venugopal, P. D., and Finkenbinder, C. (2016). Field-evolved resistance in corn earworm to Cry proteins expressed by transgenic sweet corn. PloS One 11, e0169115. doi: 10.1371/journal.pone.0169115

Dolezal, A. L., Obrian, G. R., Nielsen, D. M., Woloshuk, C. P., Boston, R. S., and Payne, G. A. (2013). Localization, morphology and transcriptional profile of Aspergillus flavus during seed colonization. Mol. Plant Pathol. 14, 898-909. doi: $10.1111 / \mathrm{mpp} .12056$

Döll, K., Chatterjee, S., Scheu, S., Karlovsky, P., and Rohlfs, M. (2013). Fungal metabolic plasticity and sexual development mediate induced resistance to arthropod fungivory. Proc. R. Soc. B.: Biol. Sci. 280. doi: 10.1098/ rspb.2013.1219 20131219.

Dowd, P. F. (1988). Toxicological and biochemical interactions of the fungal metabolites fusaric acid and kojic acid with xenobiotics in Heliothis zea (F.) and Spodoptera frugiperda (J.E. Smith). Pesticide Biochem. Physiol. 32, 123-134. doi: 10.1016/0048-3575(88)90005-3

Dowd, P. F. (2000). Indirect Reduction of Ear Molds and Associated Mycotoxins in Bacillus thuringiensis Corn Under Controlled and Open Field Conditions:
Utility and Limitations. J. Econ. Entomol. 93, 1669-1679. doi: 10.1603/00220493-93.6.1669

Drakulic, J., Caulfield, J., Woodcock, C., Jones, S. P. T., Linforth, R., Bruce, T. J. A., et al. (2015). Sharing a host plant (wheat Triticum aestivum) increases the fitness of Fusarium graminearum and the severity of Fusarium head blight but reduces the fitness of grain aphids (Sitobion avenae). Appl. Environ. Microbiol. 81, 3492-3501. doi: 10.1128/AEM.00226-15

Drakulic, J., Ajigboye, O., Swarup, R., Bruce, T., and Ray, R. V. (2016). Aphid Infestation Increases Fusarium langsethiae and T-2 and HT-2 Mycotoxins in Wheat. Appl. Environ. Microbiol. 82, 6548-6556. doi: 10.1128/AEM.02343-16

Drott, M. T., Lazzaro, B. P., Brown, D. L., Carbone, I., and Milgroom, M. G. (2017). Balancing selection for aflatoxin in Aspergillus flavus is maintained through interference competition with, and fungivory by insects. Proc. Biol. Sci. 284 (1869), 20172408. doi: 10.1098/rspb.2017.2408

Guo, B., Ji, X., Ni, X., Fountain, J. C., Li, H., Abbas, H. K., et al. (2017). Evaluation of maize inbred lines for resistance to pre-harvest aflatoxin and fumonisin contamination in the field. Crop J. 5, 259-264. doi: 10.1016/j.cj.2016.10.005

Gupta, A., and Gopal, M. (2002). Aflatoxin production by Aspergillus flavus isolates pathogenic to coconut insect pests. World J. Microbiol. Biotechnol. 18, 329. doi: 10.1023/A:1015200311512

Handley, D. T. (2018). Major Corn Earworm Flight Threatens Late Corn. Sweet Corn IPM Newsletter-September 6, No. 11 (Orono: University of Maine). Available at: https://extension.umaine.edu/highmoor/blog/tag/pestmanagement/.

Henry, W. B., Williams, W. P., Windham, G. L., and Hawkins, L. K. (2009). Evaluation of maize inbred lines for resistance to Aspergillus and Fusarium ear rot and mycotoxin accumulation. Agron. J. 101, 1219-1226. doi: 10.2134/ agronj2009.0004

Henry, W. B. (2013). Maize aflatoxin accumulation segregates with early maturing selections from an S2 breeding cross population. Toxins 5, 162-172. doi: 10.3390/toxins5010162

Herbert, A., Hull, H., and Day, E. (2003). Corn Earworm Biology and Management in Soybeans (Virginia Cooperative Extension publication) (Blacksburg: Virginia Tech). Available at: https://vtechworks.lib.vt.edu/bitstream/handle/10919/50287/ 444-770.pdf? sequence=1.

Hung, H.-Y., Shannon, L. M., Tian, F., Bradbury, P. J., Chen, C., Flint-Garcia, S. A., et al. (2012). ZmCCT and the genetic basis of day-length adaptation underlying the postdomestication spread of maize. Proc. Natl. Acad. Sci. 109, E1913E1921. doi: 10.1073/pnas.1203189109

Hutchison, W. D., Burkness, E. C., Mitchell, P. D., Moon, R. D., Leslie, T. W., Fleischer, S. J., et al. (2010). Areawide suppression of European Corn Borer with Bt maize reaps savings to non-Bt maize growers. Science 330, 222-225. doi: $10.1126 /$ science. 1190242

Johnson, M. W., Stinner, R. E., and Rabb, R. L. (1975). Ovipositional response of Heliothis zea (Boddie) to its major hosts in North Carolina. Environ. Entomol. 4, 291-297. doi: 10.1093/ee/4.2.291

Kaur, G., Guo, J., Brown, S., Head, G. P., Price, P. A., Paula-Moraes, S., et al. (2019). Field-evolved resistance of Helicoverpa zea (Boddie) to transgenic maize expressing pyramided Cry1A.105/Cry2Ab2 proteins in northeast Louisiana, the United States. J. Invertebrate Pathol. 163, 11-20. doi: 10.1016/ j.jip.2019.02.007

Lee, L. S., Lillehoj, E. B., and Kwolek, W. F. (1980). Aflatoxin distribution in individual com kernels from intact ears. Cereal Chem. 57, 340-343.

Lillehoj, E. B., McMillian, W. W., Widstrom, N. W., Guthrie, W. D., Jarvis, J. L., Barry, D., et al. (1984). Aflatoxin contamination of maize kernels before harvest: Interaction of Aspergillus flavus spores, corn earworm larvae and fungicide applications. Mycopathologia 86, 77-81. doi: 10.1007/BF00436490

Lopez-garcia, R. (1998). Aflatoxin B(1) and Fumonisin B(1) co-contamination: Interactive effects, possible mechanisms of toxicity, and decontamination procedures. LSU Historical Dissertations and Theses. (Baton Rouge: Louisiana State University) 6746.

Madege, R. R., Audenaert, K., Kimanya, M., Tiisekwa, B., De Meulenaer, B., Bekaert, B., et al. (2018). Control of Fusarium verticillioides (Sacc.) Nirenberg and Fumonisins by Using a Combination of Crop Protection Products and Fertilization. Toxins 10, 67. doi: 10.3390/toxins 10020067

Mesterházy, Á, Lemmens, M., and Reid, L. M. (2012). Breeding for resistance to ear rots caused by Fusarium spp. in maize - a review. Plant Breed. 131, 1-19. doi: 10.1111/j.1439-0523.2011.01936.x 
Mideros, S. X., Windham, G. L., Williams, W. P., and Nelson, R. J. (2012). Tissuespecific components of resistance to Aspergillus ear rot of maize. Phytopathology 102, 787-793. doi: 10.1094/PHYTO-12-11-0355

Miller, J. D. (1994). "Epidemiology of Fusarium ear diseases of cereals," in Mycotoxins in Grain: Compounds Other Than Aflatoxin, 1st ed. Eds. J. D. Miller and H. L. Trenholm (St. Paul, MN, USA.: Eagan Press), 19-36.

Morales, L., Zila, C. T., Moreta Mejía, D. E., Montoya Arbelaez, M., Balint-Kurti, P. J., Holland, J. B., et al. (2019). Diverse components of resistance to Fusarium verticillioides infection and fumonisin contamination in four maize recombinant inbred families. Toxins 11, 86. doi: 10.3390/toxins 11020086

Munkvold, G. P., and White, D. G. (2016). Compendium of corn diseases. 4th ed. (St. Paul, Minnesota, USA: The American Phytopathological Society).

Munkvold, G. P., Arias, S., Taschl, I., and Gruber-Dorninger, C. (2019). "Chapter 9 - Mycotoxins in Corn: Occurrence, Impacts, and Management," in Corn, 3rd ed. Ed. S. O. Serna-Saldivar (Oxford: AACC International Press), p 235-p 287. Available at: https://doi.org/10.1016/B978-0-12-811971-6.00009-7.

Munkvold, G. P. (2003). Epidemiology of Fusarium Diseases and their Mycotoxins in Maize Ears. Eur. J. Plant Pathol. 109, 705-713. doi: 10.1023/ A:1026078324268

Ni, X., Wilson, J. P., Buntin, G. D., Guo, B., Krakowsky, M. D., Lee, R. D., et al. (2011). Spatial Patterns of Aflatoxin Levels in Relation to Ear-Feeding Insect Damage in Pre-Harvest Corn. Toxins 3, 920. doi: 10.3390/toxins3070920

Opoku, J., Kleczewski, N. M., Hamby, K. A., Herbert, D. A., Malone, S., and Mehl, H. L. (2019). Relationship between invasive Brown Marmorated Stink Bug (Halyomorpha halys) and fumonisin contamination of field corn in the mid-Atlantic U.S. Plant Dis. 103, 1189-1195. doi: 10.1094/PDIS-06-181115-RE

Piesik, D., Lemńczyk, G., Skoczek, A., Lamparski, R., Bocianowski, J., Kotwica, K., et al. (2011). Fusarium infection in maize: Volatile induction of infected and neighboring uninfected plants has the potential to attract a pest cereal leaf beetle, Oulema melanopus. J. Plant Physiol. 168, 1534-1542. doi: 10.1016/ j.jplph.2011.01.032

Plattner, R. D. (1999). HPLC/MS analysis of Fusarium mycotoxins, fumonisins and deoxynivalenol. Natural Toxins 76, 365-370. doi: 10.1002/1522-7189 (199911/12)7:6<365::AID-NT85>3.0.CO;2-0

Riley, R. T., and Merrill, A. H. (2019). Ceramide synthase inhibition by fumonisins: a perfect storm of perturbed sphingolipid metabolism, signaling and disease. J. Lipid Res. 60, 1183-1189. doi: 10.1194/jlr.S093815

Riley, R. T., Enongene, E., Voss, K. A., Norred, W. P., Meredith, F. I., Sharma, R. P., et al. (2001). Sphingolipid perturbations as mechanisms for fumonisin carcinogenesis. Environ. Health Perspect. 109 Suppl 2, 301-308. doi: 10.1289/ ehp.01109s2301

Santiago, R., Cao, A., and Butrón, A. (2015). Genetic factors involved in fumonisin accumulation in maize kernels and their implications in maize agronomic management and breeding. Toxins 7, 3267-3296. doi: 10.3390/ toxins 7083267

Schuh, M., and Springborn, F. (2019). Michigan State University Extension, July 31, 2019. (East Lansing: Michigan State University) Available at: https://www. canr.msu.edu/news/managing-corn-earworm-in-an-unusual-year.

Schulthess, F., Cardwell, K. F., and Gounou, S. (2002). The effect of endophytic Fusarium verticillioides on infestation of two maize varieties by Lepidopterous stemborers and Coleopteran grain feeders. Phytopathology 92, 120-128. doi: 10.1094/PHYTO.2002.92.2.120

Scott, G. E., and Zummo, N. (1990). Registration of Mp313E Parental Line of Maize. Crop Sci. 30, 1378-1378. doi: 10.2135/cropsci1990.0011183X003000060080x

Shirima, C. P., Kimanya, M. E., Kinabo, J. L., Routledge, M. N., Srey, C., Wild, C. P., et al. (2013). Dietary exposure to aflatoxin and fumonisin among Tanzanian children as determined using biomarkers of exposure. Mol. Nutr. Food Res. 57, 1874-1881. doi: 10.1002/mnfr.201300116
Shu, X., Livingston, D. P., Franks, R. G., Boston, R. S., Woloshuk, C. P., and Payne, G. A. (2014). Tissue-specific gene expression in maize seeds during colonization by Aspergillus flavus and Fusarium verticillioides. Mol. Plant Pathol. 16, 662-674. doi: 10.1111/mpp.12224

Smeltzer, D. (1959). Relationship between Fusarium ear rot and corn earworm infestation. Agron. J. 51, 53-55. doi: 10.2134/agronj1959.00021962005100010019x

St Leger, R. J., Screen, S. E., and Shams-Pirzadeh, B. (2000). Lack of host specialization in Aspergillus flavus. Appl. Environ. Microbiol. 66, 320-324. doi: 10.1128/AEM.66.1.320-324.2000

Sun, G., Wang, S., Hu, X., Su, J., Zhang, Y., Xie, Y., et al. (2011). Co-contamination of aflatoxin $\mathrm{B} 1$ and fumonisin $\mathrm{B} 1$ in food and human dietary exposure in three areas of China. Food Addit. Contamin.: Part A. 28, 461-470. doi: 10.1080/ 19440049.2010.544678

TePaske, M. R., Gloer, J. B., Wicklow, D. T., and Dowd, P. F. (1992). Aflavarin and $\beta$-aflatrem: new anti-insectan metabolites from the sclerotia of Aspergillus flavus. J. Natural Prod. 55, 1080-1086. doi: 10.1021/np50086a008

Venugopal, P. D., and Dively, G. P. (2017). Climate change, transgenic corn adoption and field-evolved resistance in corn earworm. R. Soc. Open Sci. 4, 170210. doi: $10.1098 /$ rsos. 170210

Widstrom, N. W. (1992). "Aflatoxin in developing maize: Interactions among involved biota and pertinent econiche factors," in Handbook of Applied Mycology Vol. 5, Mycotoxins in Ecological Systems. Ed. D. Bhatnagar, E. B. Lillehoj and D. K. Arora (Basel: Marcel Dekker Inc.). pp. 59-86.

Williams, W. P., and Windham, G. L. (2006). Registration of Maize Germplasm Line Mp717 Joint contribution of USDA-ARS and the Mississippi Agricultural and Forestry Experiment Station. Miss. Agric. and Forestry Exp. Stn. Journal No. J-10802. Registration by CSSA. Crop Sci. 46, 1407-1408. doi: 10.2135/ cropsci2005.09-0330

Williams, W. P., and Windham, G. L. (2009). Diallel analysis of fumonisin accumulation in maize. Field Crops Res. 114, 324-326. doi: 10.1016/ j.fcr.2009.08.005

Williams, W. P. (2006). Breeding for resistance to aflatoxin accumulation in maize. Mycot. Res. 22, 27-32. doi: 10.1007/BF02954554

Windham, G. L., Williams, W. P., Mylroie, J. E., Reid, C. X., and Womack, E. D. (2018). A Histological study of Aspergillus flavus colonization of wound inoculated maize kernels of resistant and susceptible maize Hybrids in the field. Front. Microbiol. 9, 799-799. doi: 10.3389/fmicb.2018.00799

World Health Organization (2018). Co-exposure of fumonisins with aflatoxins. Food Safety Digest (Geneva: World Health Organization). WHO/NHM/FOS/ RAM/18.3. doi: 10.2135/cropsci2005.09-0330

Zeng, R. S. L., Niu, G., Wen, Z., Schuler, M. A., and Berenbaum, M. R. (2006). Toxicity of Aflatoxin B1 to Helicoverpa zea and Bioactivation by Cytochrome P450 Monooxygenases. J. Chem. Ecol. 32, 1459-1471. doi: 10.1007/s10886006-9062-7

Zummo, N., and Scott, G. E. (1992). Interaction of Fusarium moniliforme and Aspergillus flavus on Kernel Infection and Aflatoxin Contamination in Maize Ears. Plant Dis. 76, 771-773. doi: 10.1094/PD-76-0771

Conflict of Interest: The authors declare that the research was conducted in the absence of any commercial or financial relationships that could be construed as a potential conflict of interest.

Copyright $\odot 2020$ Chalivendra, Huang, Busman, Williams and Ham. This is an openaccess article distributed under the terms of the Creative Commons Attribution License (CC BY). The use, distribution or reproduction in other forums is permitted, provided the original author(s) and the copyright owner(s) are credited and that the original publication in this journal is cited, in accordance with accepted academic practice. No use, distribution or reproduction is permitted which does not comply with these terms. 